\title{
Intratracheal Versus Intravenous Liposomal Delivery of siRNA, Antisense Oligonucleotides and Anticancer Drug
}

\author{
Olga B. Garbuzenko, ${ }^{1}$ Maha Saad, ${ }^{1}$ Seema Betigeri, ${ }^{1}$ Min Zhang, ${ }^{1}$ Alexandre A. Vetcher, ${ }^{2,5}$ \\ Viatcheslav A. Soldatenkov, ${ }^{3,6}$ David C. Reimer, ${ }^{4}$ Vitaly P. Pozharov, ${ }^{1}$ and Tamara Minko ${ }^{1,7}$
}

Received June 1, 2008; accepted October 10, 2008; published online October 29, 2008

Purpose. To compare systemic intravenous and local intratracheal delivery of doxorubicin (DOX) antisense oligonucleotides (ASO) and small interfering RNA (siRNA).

Methods. "Neutral" and cationic liposomes were used to deliver DOX, ASO, and siRNA. Liposomes were characterized by dynamic light scattering, zeta-potential, and atomic force microscopy. Cellular internalization of DOX, ASO and siRNA was studied by confocal microscopy on human lung carcinoma cells. In vivo experiments were carried out on nude mice with an orthotopic model of human lung cancer. Results. Liposomes provided for an efficient intracellular delivery of DOX, ASO, and siRNA in vitro. Intratracheal delivery of both types of liposomes in vivo led to higher peak concentrations and much longer retention of liposomes, DOX, ASO and siRNA in the lungs when compared with systemic administration. It was found that local intratracheal treatment of lung cancer with liposomal DOX was more efficient when compared with free and liposomal DOX delivered intravenously.

Conclusions. The present study outlined the clear advantages of local intratracheal delivery of liposomal drugs for the treatment of lung cancer when compared with systemic administration of the same drug.

KEY WORDS: antisense oligonucleotides; imaging; liposomes; local lung delivery of siRNA; lung cancer; pulmonary delivery.

\section{INTRODUCTION}

Every year, close to 400,000 Americans die of lung disease, making it the third most frequent cause of death in the USA (one in seven deaths). An additional 35 million Americans are living with chronic lung diseases (1). Therefore, the development of effective methods of drug delivery

\footnotetext{
${ }^{1}$ Department of Pharmaceutics, Ernest Mario School of Pharmacy, Rutgers, The State University of New Jersey, 160 Frelinghuysen Road, Piscataway, New Jersey 08854-8020, USA.

${ }^{2}$ National Center for Biodefense and Infectious Diseases, George Mason University, Manassas, Virginia, USA.

${ }^{3}$ Department of Radiation Medicine, Lombardi Comprehensive Cancer Center, Georgetown University Medical Center, Washington, District of Columbia, USA.

${ }^{4}$ Laboratory Animal Services, Rutgers, The State University of New Jersey, Piscataway, New Jersey, USA.

${ }^{5}$ Alcyon Bio, Inc., Germantown, Maryland 20874, USA.

${ }^{6}$ DEA, SRLB, National Cancer Institute, National Institutes of Health, Bethesda, Maryland, USA.

${ }^{7}$ To whom correspondence should be addressed. (e-mail: minko@rci. rutgers.edu)

ABBREVIATIONS: AFM, Atomic force microscopy; ASO, Antisense oligonucleotides; DOTAP, 1,2-Dioleoyl-3-trimethylammonium-propane; DOX, Doxorubicin; DSPE-PEG, 1,2-Distearoyl-sn-glycero-3phosphoetanol amine- $N$-aminopolyethylene glycol- $M_{\mathrm{w}} \sim 2000$ ammonium salt; FITC, Fluorescein isothiocyanate; i.t, Intratracheal; i.v., Intravenous; PEG, Poly(ethylene glycol); siRNA, Small interfering RNA; RES, Reticuloendothelial system.
}

to the lungs is an immediate and important task of modern medicine. Traditionally, three main routes of administration are being used to deliver therapeutic ingredients to the lungs: oral, intravenous, and pulmonary. After oral administration, drugs suffer from the destruction in gastro-intestinal tract and during first-pass liver metabolism. Drugs delivered via an intravenous route of administration accumulate not only in the lungs but also in other organs $(2,3)$ and may undergo degradation in the systemic circulation. Local drug delivery to the lung may enhance drug retention by lung cells, increase drug concentration inside the cells, decrease the need for high drug dose, and limit the adverse side effects of the treatment on healthy organs. Liposomes, small lipid vesicles with a composition close to lung surfactant, have been successfully used for the delivery of different therapeutic agents to the lung (4-11). However, distribution of different types of liposomal carriers and their payloads through the body after the inhalation as well as their ability to deliver diverse active ingredients inside lung cells have not been studied in detail. These aspects are especially important when liposomes are used for the delivery of potentially toxic ingredients (antibiotics, anticancer drugs, etc.) and/or other highly effective therapeutic components (proteins, antisense oligonucleotides, DNA, RNA, etc).

Liposomes have been widely investigated since 1970 as drug carriers for improving the delivery of therapeutic agents to specific sites in the body and almost immediately were explored for cancer treatment $(12,13)$. One of the serious obstacles limiting therapeutic applications of liposomes is short circulation residence time as a result of their elimination from the blood 
stream by reticuloendothelial system (RES) after systemic administration $(14,15)$. This problem was partially solved by covering the liposome surface with phosphoetanolamine derivatives of poly(ethylene glycol) (PEG) or other polymers. These "neutral" and sterically stabilized liposomes (Stealth liposomes) used as carriers for hydrophilic anticancer drugs (doxorubicin, vincristine etc.) exhibited really long circulation time in the vascular system (16-19) and showed enhanced accumulation of drugs in tumor tissues (20-23).

During the past 20 years, a variety of techniques have been developed to encapsulate and/or deliver therapeutics with liposomes. Liposomes have been used successfully to deliver conventional drugs or new genetic drugs including plasmid DNA-containing therapeutic genes, antisense oligonucleotides (ASO), and small interfering RNA (siRNA) in preclinical models and clinical trials (24-32). The delivery of different types of payloads in turn requires different properties of carriers, including their surface charge. Lipophilic and hydrophilic drugs can be incorporated into the lipid membrane or inner aqueous space of liposomes respectively. While modified uncharged nucleotides can be delivered by neutral or slightly charged liposomes, native negatively charged ASO, siRNA, or DNA molecules required cationic liposomes. The practical application of different types of liposomes for inhalatory delivery of various therapeutic components is limited by the almost complete absence of data describing organ exposures by different liposomes after their local intratracheal delivery.

In the present study, traditional "neutral" PEGylated and 1,2-dioleoyl-3-trimethylammonium-propane (DOTAP) cationic liposomes were investigated. DOX and neutral Pethoxy-ASO were used as payloads for "neutral" liposomes while DOTAP was used to deliver negatively charged siRNA. The study was carried out to fulfill three specific aims. First, internalization of liposomes with different sizes and charges into lung carcinoma cells was compared in vitro. Second, two distinct routes of administration, systemic intravenous and local intratracheal, were compared in vivo on mice using similar "neutral" and cationic liposomes with respective payload. Third, the effectiveness of intravenous and intratracheal treatment of lung cancer with liposomal DOX were compared on nude mice with an orthotopic model of human lung human A549 non-small cell lung carcinoma.

\section{MATERIALS AND METHODS}

Cell Line. A549-luc-C8 light producing cell line derived from A549 human non-small cell lung carcinoma cells by stable transfection of the North American Firefly Luciferase gene expressed from the CMV promoter was obtained from Xenogen Corporation (Alameda, California). Cells were cultured in RPMI medium, supplemented with $10 \%$ fetal bovine serum (Fisher Chemicals, Fairlawn, NJ). Cells were grown at $37^{\circ} \mathrm{C}$ in a humidified atmosphere of $5 \% \mathrm{CO}_{2}(v / v)$ in air. All experiments were performed on cells in the exponential growth phase.

Animals. Athymic nu/nu mice 6-8 weeks old were obtained from Taconic (Hudson, NY). All mice were maintained in micro-isolated cages under pathogen free conditions in the animal maintenance facilities of Rutgers, The State University of New Jersey.
Liposomal Compositions of Doxorubicin, Antisense Oligonucleotides and siRNA. PEGylated "neutral" liposomes were used for delivery of electrically neutral DOX and P-ethoxy ASO. Negatively charged siRNA was delivered by cationic DOTAP liposomes. ASO targeted to BCL2 mRNA were synthesized and labeled with fluorescein isothiocyanate (FITC) by Oligos Etc. (Wilsonville, OR) according to our design (29). The DNA backbone of all bases in oligonucleotides was P-ethoxy modified to eliminate an electrical charge and increase incorporation efficacy into "neutral" liposomes. A siGLO Red transfection indicator (RNA duplex labeled with Pierce NuLight DY-547 fluorophore) was purchased from Dharmacon Inc. (Chicago, IL) and was used to study siRNA delivery and cellular uptake. Doxorubicin was purchased from Sigma (St. Louis, MO).

To prepare "neutral" liposomes, lipids (Avanti Polar Lipids, Alabaster, AL) were dissolved in chloroform, evaporated to a thin film layer in a rotary evaporator, and rehydrated with $300 \mathrm{mM}$ citrate buffer, $\mathrm{pH}$ 4. The lipid mole ratio for this formulation was 51:44:5 egg phosphatidylcholin/ cholesterol/1,2,-distearoyl-sn-glycero-3-phosphoethanolamine- $N$-aminopolyethelenglycol- $M_{\mathrm{w}}-2000$ ammonium salt respectively. ASO was loaded into liposomes by dissolving in rehydration buffer in concentration $0.5 \mathrm{mM}$. Liposomes were extruded gradually using polycarbonate membranes 200 and $100 \mathrm{~nm}$ at room temperature using an extruder device from Northern Lipids, Inc. (Vancouver, BC, Canada). Obtained liposomes were dialyzed overnight against $0.9 \% \mathrm{NaCl}$ at $4^{\circ} \mathrm{C}$, and loaded with DOX $(5 \mathrm{mg} / \mathrm{mL})$ at $37^{\circ} \mathrm{C}$. Liposomes were separated from free DOX by dialysis against 100 volumes $0.9 \%$ $\mathrm{NaCl}$. The encapsulation efficacy of DOX and ASO was $\sim 95 \%$ and $\sim 50 \%$ respectively. The final phospholipids concentration was $10 \mathrm{mg} / \mathrm{mL}$. Mean liposomes diameter was $100-140 \mathrm{~nm}$. Cationic liposomes were prepared from positively charged dioleoyl-2-trimethylammonium propane-cationic (Avanti Polar Lipids, Alabaster, AL) in concentration $5 \mathrm{mg} / \mathrm{mL}$ using thin layer procedure described above and followed by extrusion through $100 \mathrm{~nm}$ polycarbonate membrane. siGLO Red was dissolved in RNAse free solution to final concentration $200 \mu \mathrm{M}$. DOTAP liposomes were mixed with siGLO in ratio 6:1 v/v and incubated at room temperature for $15 \mathrm{~min}$ before use. Mean DOTAP/ siGLO complexes size was $>500 \mathrm{~nm}$.

Aliquots of each liposomal formulation were labeled with near infrared fluorescent dye Cy5.5 Mono NHS Ester (GE Healthcare, Amersham, UK) or fluorescein isothiocyanate FITC. A fluorescent dye was dissolved together with lipids in chlorophorm. Approximate excitation/emission maxima of all used fluorescent substances are: $675 \mathrm{~nm} /$ $694 \mathrm{~nm}$ (Cy5.5); $557 \mathrm{~nm} / 570 \mathrm{~nm}$ (siGLO Red, siRNA labeled with DY-547 dye); $494 \mathrm{~nm} / 518 \mathrm{~nm}$ (FITC); $470 \mathrm{~nm} /$ $585 \mathrm{~nm}(\mathrm{DOX})$.

Liposome Size and Zeta Potential. Particle size was measured by dynamic light scattering using 90 Plus Particle Sizer Analyzer (Brookhaven Instruments Corp., New York, $\mathrm{NY}$ ). Aliquot of $40 \mu \mathrm{L}$ of each sample was diluted in $2 \mathrm{~mL}$ of its external buffer. Zeta potential was measured on PALS Zeta Potential Analyzer (Brookhaven Instruments Corp, New York, NY). Samples were taken as is and their volume was $1.5 \mathrm{~mL}$. All measurements were carried out at room temperature. Each parameter was measured in triplicate, and average values were calculated. 
Atomic Force Microscopy. The shape of cationic, "neutral" conventional and PEGylated liposomes were studied by atomic force microscopy (AFM) imaging using the previously described procedure (33). Briefly, $50 \mu \mathrm{L}$ of liposome suspension in water was deposited on pre-cut $\left(\sim 25 \times 25 \mathrm{~mm}^{2}\right)$ and pre-cleaned Plain Premium microscope slides (Fisher Scientific Co, Pittsburgh, PA), kept for $10 \mathrm{~min}$ at $100 \%$ humidity to achieve particles precipitation. Water was removed by dry nitrogen flow and dried samples were subjected to imaging with an atomic force microscope (Nano-R AFM Pacific Nanotechnology Instrument, PNI, Santa Clara, CA) in close contact (tapping) mode using tapping mode etched OMCLAC160TS silicon probes (Olympus Optical Co. Tokyo, Japan). The captioning was performed in the height mode. The images were processed, and the measurements were performed with Femtoscan software v. 2.2.85(5.1) (Advanced Technologies Center, Moscow, Russia). For statistics, no less than 50 objects of each sample were analyzed.

Confocal Microscopy. Cellular internalization of liposomes, DOX, ASO and siRNA were monitored in A549 lung cancer cells by confocal microscopy. Three series of the experiments were carried out: (1) FITC-labeled "neutral" liposomes (green fluorescence) contained DOX (intrinsic red fluorescence); (2) "neutral" liposomes labeled with Cy5.5 (red fluorescence) contained ASO labeled with FITC (green fluorescence); (3) FITC-labeled cationic liposomes (green fluorescence) contained siGLO Red (red fluorescence). Cells were separately incubated $24 \mathrm{~h}$ at $37^{\circ} \mathrm{C}$ with each liposomal formulation. Red and green fluorescence images were photographed and digitally overlaid. Superimposition of images allows for detecting of co-localization of labeled liposomes and their payload (yellow color).

Content of Liposomes in Different Organs. The distribution of different liposomes and their payload was examined on nude mice. Labeled, empty "neutral" and cationic liposomes as well as different liposomes containing DOX, labeled ASO or siRNA were delivered by intratracheal or intravenous administrations. Injected volume of liposomes was $100 \mu \mathrm{L}$ for each formulation; DOX concentration was $2 \mathrm{mg} / \mathrm{kg}$. Animals were anesthetized with isoflurane and euthanized $30 \mathrm{~min}, 1 \mathrm{~h}$, and $24 \mathrm{~h}$ after the treatment. Lungs, heart, liver, spleen, and kidneys were excised, rinsed in saline, and fluorescence was registered by IVIS imaging system (Xenogen Corporation, Alameda, CA). Visible light and fluorescence images were taken and overlaid. The intensity of fluorescence was represented on composite light/fluorescent images by different colors with blue color reflecting the lowest fluorescence intensity and red color-the highest intensity. Images of each organ were then scanned and total fluorescence intensity was calculated by a special computer program originally developed for our laboratory by Dr. V. P. Pozharov. Preliminary experiments showed a strong linear correlation between the total amount of labeled substance accumulated in the organ and calculated total fluorescence intensity (data are not shown). The fluorescence was expressed in arbitrary units with 1 units represented approximately $2 \times 10^{10}$ photons $/ \mathrm{s} / \mathrm{sr} / \mathrm{cm}^{2}$. The method allows a quantitative comparison of the concentration of the same fluorescent dye between different series of the experiments. The mass of all organs was measured. No statistical differences were found in the mass of each organ between all experimental groups of animals. The fluorescence intensity was normalized for organ weight. In an additional series of the experiments, fluorescence of labeled "neutral" and cationic liposomes in lungs was analyzed $30 \mathrm{~min}, 1 \mathrm{~h}, 1$ day, 3 days, 5 days, 8 days, and 10 days after the administration. The concentration of liposomes in the lungs was plotted versus the time, and area under the curve (AUC) reflecting a total lung tissue exposure was calculated.

Orthotopic Mouse Model of Human Lung Cancer and Tumor Treatment. Orthotopic engraftment of tumor was established by intratracheal (i.t.) instillation of $5.0 \times 10^{6}$ A549 human non-small cell lung carcinoma cells into athymic $n u / n u$ mice. Tumor growth was monitored once a week by real-time bioluminescence in vivo with an imaging system using luciferase expressing lung cancer cells (Xenogen Bioscience, Cranbury, NJ). To compare the anticancer effectiveness of local (intratracheal) and systemic (intravenous) administration of liposomal drugs, the following experiments have been performed. After the instillation of cancer cells, tumor growth was monitored for 6 weeks and mice were randomized into four groups (five animals per group) based on tumor burden as measured by in vivo imaging. The animals from the first group were not treated (untreated tumor control). Group 2 received a single dose of doxorubicin $(2 \mathrm{mg} / \mathrm{kg})$ via intravenous injection in phosphate buffered saline. Groups 3 and 4 received the liposomal formulation of doxorubicin $(2 \mathrm{mg} / \mathrm{kg})$ via intravenous injection and intratracheal instillation, respectively. The dose of $2 \mathrm{mg} / \mathrm{kg}$ was estimated in preliminary experiments as a maximum tolerated dose for liposomal DOX. Twenty-four hours after the treatment, animals from all groups were sacrificed, and lung tissue samples from each animal were fixed in $10 \%$ buffered formalin. Each tissue sample was trimmed, embedded in paraffin, sectioned and stained with hematoxylin and eosin for histopathological evaluation. The evaluation was performed by a veterinary pathologist. Ten slices per each sample were evaluated under the same magnification. The number of cancer cells in each microscopic field of view was calculated and presented as percent of untreated tumor control (group \# 2).

Endotracheal Instillation of Lung Cancer Cells and Liposomes. Mice were anesthetized through intraperitoneal injection with $80 \mathrm{mg} / \mathrm{kg}$ ketamine and $10-12 \mathrm{mg} / \mathrm{kg}$ xylazine. Once anesthetized, the mouse was placed on the titling rodent work stand (Hallowell EMC, Pittsfield, MA) in supine position and restrained in position by an incisor loop. The tongue was then extruded using atraumatic forceps or via rotation with a cotton tip applicator. The larynx was visualized using a modified $4 \mathrm{~mm}$ ear speculum attached to an operating head of an ophthalmoscope (Welch Allyn, Skaneateles Falls, NY). The modified speculum, acting as a laryngoscope blade, in an inverted fashion provided dorsal displacement of the tongue and magnification of the laryngeal opening. One hundred microliters of cells or liposomes in suspension were instilled within the trachea using a $40 \mathrm{~mm}$ length of Clay Adams Intramedic polyethylene tubing (Diagnostic Systems, Sparks, MD, PE-10, I.D $0.28 \mathrm{~mm}$, O.D. $0.61 \mathrm{~mm}$ ), attached to a 27 gauge hypodermic needle. The tubing was advanced approximately $10 \mathrm{~mm}$ past the epiglottis. The tubing and speculum were withdrawn immediately after instillation. The mouse was then 
Table I. Size and Zeta Potential of Liposomes at Room Temperature

\begin{tabular}{lcc}
\hline \multicolumn{1}{c}{ Liposomes/parameter } & Size $(\mathrm{nm})^{a}$ & $\begin{array}{l}\text { Zeta potential } \\
(\mathrm{mV})^{a}\end{array}$ \\
\hline EPC/Chol/DSPE-PEG & $120 \pm 20$ & $-10 \pm 2$ \\
$\begin{array}{l}\text { EPC/Chol/DSPE-PEG loaded } \\
\quad \text { with DOX }\end{array}$ & $130 \pm 10$ & $-10 \pm 2$ \\
$\begin{array}{l}\text { EPC/Chol/DSPE-PEG loaded } \\
\quad \text { with ASO }\end{array}$ & $130 \pm 10$ & $-10 \pm 2$ \\
$\begin{array}{l}\text { DOTAP } \\
\text { DOTAP/siRNA complexes }\end{array}$ & $120 \pm 15$ & $+25 \pm 4$ \\
\end{tabular}

${ }^{a}$ Means \pm SD are shown

removed from the work stand and held in a vertical position by the scruff until multiple normal respirations were observed.

Statistical Analysis. Data were analyzed using descriptive statistics, single-factor analysis of variance (ANOVA), and presented as mean values \pm the standard deviation from four to eight independent measurements. The comparison among groups was performed by the independent sample Student's $t$ tests. The difference between variants is considered significant if $P<0.05$.

\section{RESULTS}

Particle Size and Zeta Potential Measurements. Table I shows the size and zeta potential of liposomal compositions

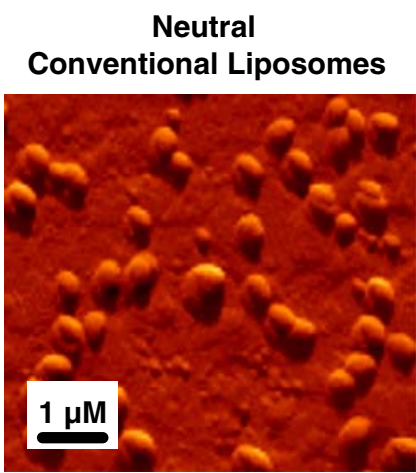

Cationic Liposomes

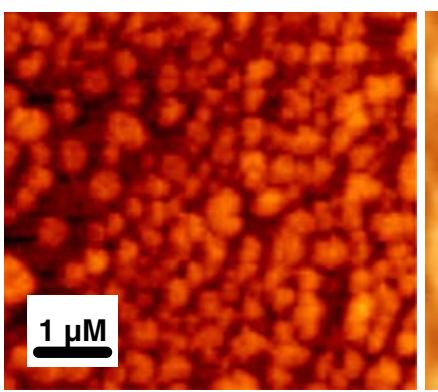

Fig. 1. Assessment of different liposomes by atomic force microscopy. Typical images of neutral conventional and "neutral" liposomes, cationic liposomes, and complexes of cationic liposomes with siRNA are shown. Liposome suspensions were applied on freshly cleaved mica, kept for $10 \mathrm{~min}$ at $100 \%$ humidity atmosphere to achieve deposition, and dried under nitrogen flow to remove external water. Panoramic views were captured in $<$ dem $>$ mode (phase contrast). used in the study. As can be seen, "neutral" liposomes have a slight negative charge. The finding that the zeta potential of lipid assemblies containing PEG-DSPE is negative is in agreement with our previously reported data (31). It also supports the "hidden charge effect" that was suggested for liposomes sterically stabilized through grafting of mPEGDSPE with PEG moiety $\geq 0.75 \mathrm{kDa}(34,35)$. Similar to their conventional counterpart, these liposomes can be considered "neutral." Inclusion of DOX and ASO into "neutral" liposomes did not change their size and zeta potential. The diameter of positively charged DOTAP liposomes after extrusion was about 100-140 nm (same as for "neutral" liposomes). Mixing with siRNA in ratio 6:1 $v / v$ lipid/siRNA respectively led to formation of rather large DOTAP/siRNA

\section{FITC-Neutral PEGylated Liposomes-DOX}

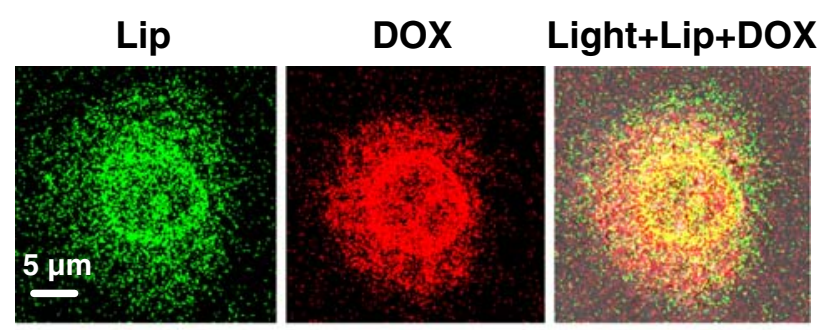

Cy5.5-Neutral PEGylated Liposomes-ASO-FITC

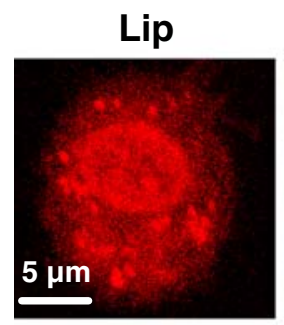

ASO
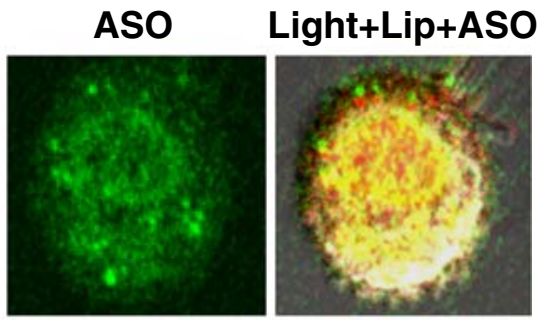

FITC-Cationic Liposomes-siRNA-DY-547 Lip

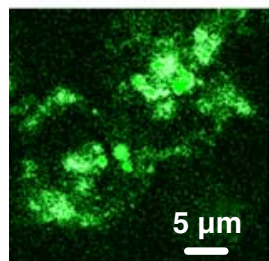
siRNA Light+Lip+siRNA
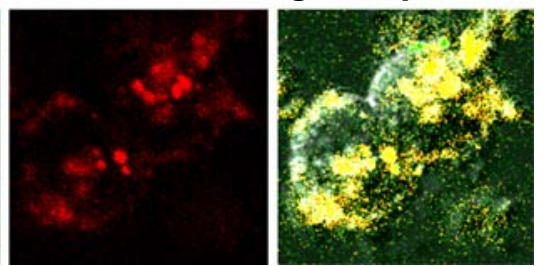

Fig. 2. Intracellular localization of different liposomes and their payload. A-549 lung cancer cells were incubated with substances indicated and visible light and fluorescent images (red and green fluorescence) were taken by a confocal microscope. Visible light image was digitally overlaid with both fluorescence images to obtain a composite image showing co-localization of liposomes and their payload (in yellow color). The upper panel contains representative images of "neutral" liposomes labeled with FITC (green fluorescence) containing Doxorubicin (DOX, intrinsic red fluorescence). The middle panel shows representative images of complexes of "neutral" liposomes labeled with near infrared dye Cy5.5 (red fluorescence) containing FITC labeled antisense oligonucleotides (ASO, green fluorescence). The bottom panel contains representative images of complexes of FITC labeled cationic liposomes (green fluorescence) with siRNA labeled with DY-547 dye (red fluorescence). Superimposition of images allows for detecting of colocalization of liposomes and their payload inside cells (yellow color). 
a

Neutral PEGylated Liposomes-Cy5.5

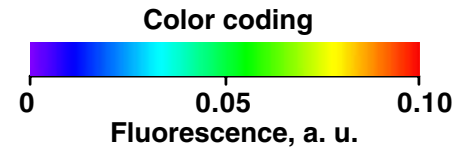

Intravenous injection

Intratracheal injections

Liver Kidneys Spleen Heart Lungs

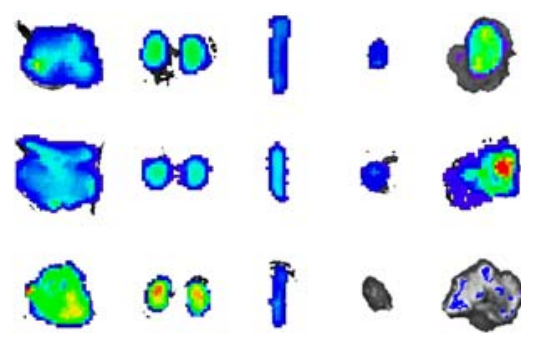

$24 \mathrm{~h}$
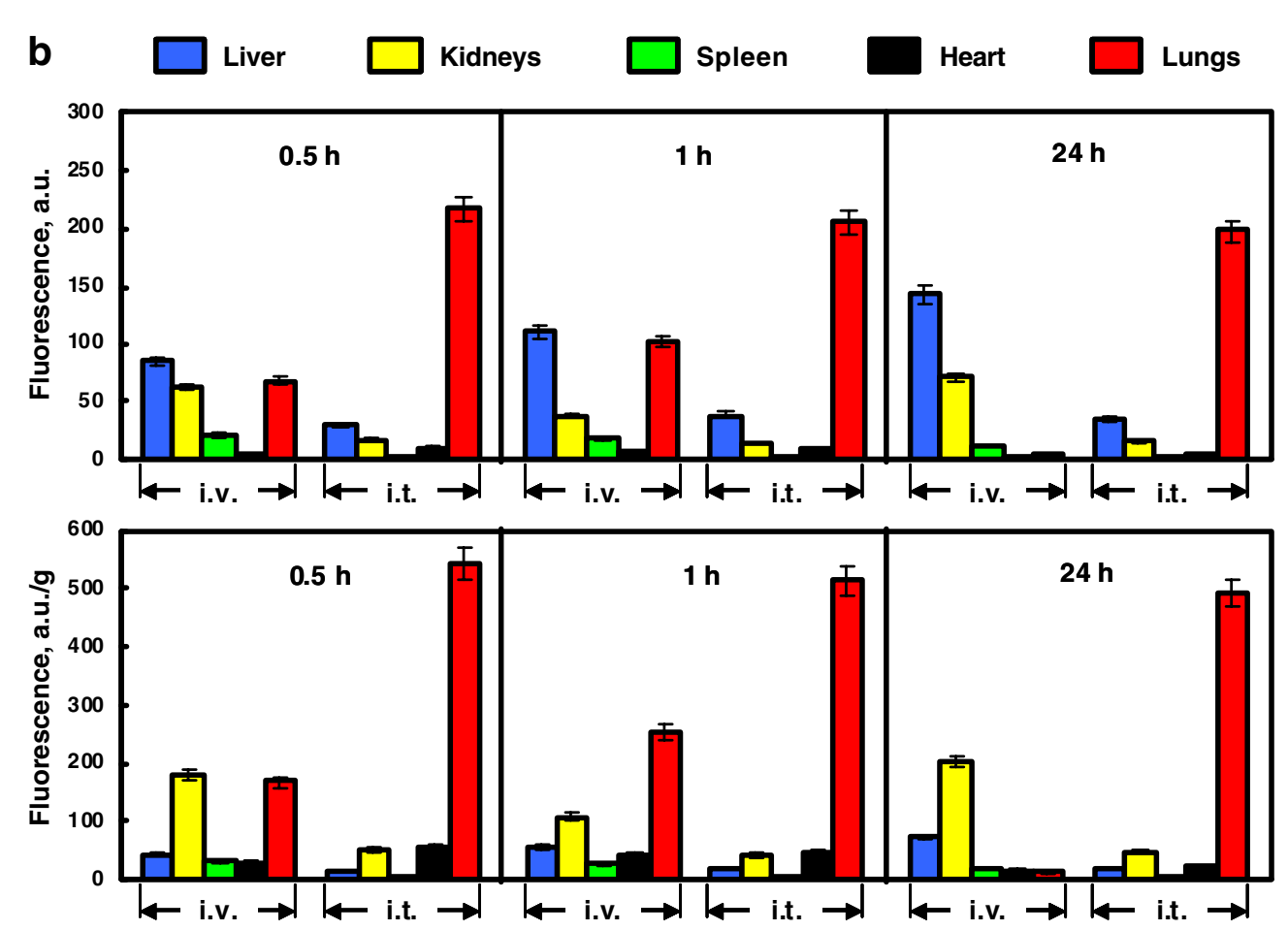

Fig. 3. Organ content of "neutral" liposomes. PEGylated liposomes were labeled with near infrared Cy5.5 dye and delivered intravenously or intratracheally into the mice. The distribution of liposomes was measured using the IVIS Xenogen imaging system. The upper panel (a) contains representative images of different organs excised $0.5,1$ and $24 \mathrm{~h}$ after injection. The intensity of fluorescence is expressed by different colors with blue color reflecting the lowest intensity and red color-highest intensity. The bottom panel (b) demonstrates average total content per an entire organ and concentration (per gram of organ weight) of labeled liposomes. Means \pm SD are shown.

complexes and decreased surface charge likely due to the electrostatic interactions between positively charged lipid and negatively charged siRNA.

Atomic Force Microscopy. Atomic force microscopy (AFM) was widely used during the last decade to characterize morphology of nanoparticles. AFM topographical images of the liposome preparations revealed convex meniscus shaped particles that were uniformly distributed on the mica surface (Fig. 1). No substantial presence of liposome aggregates were observed as expected from physico-chemical properties of uniformly charged particles. Since liposomes flatten on the mica surface after deposition and drying process results in distortion of their actual shape in suspension, we calculated the value of reconstructed diameter $(d)$ from the liposomes volume measured by AFM $(V)$ under the assumption that liposomes adopt the spherical shape in aqueous solutions, using the equation $d=(6 \mathrm{~V} / \pi)^{1 / 3}$ as previously described (31). The complexation of liposomes with siRNA resulted in more than 2.5-fold increase of their $d$ to over $500 \mathrm{~nm}$. 
a

Cationic Liposomes-Cy5.5

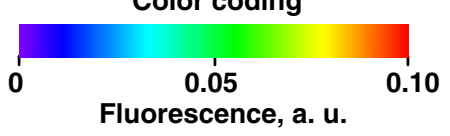

Intravenous injection

Intratracheal injections

Liver Kidneys Spleen Heart Lungs
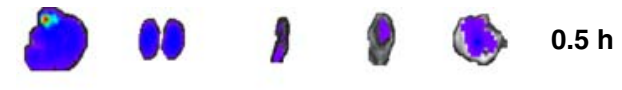

Liver Kidneys Spleen Heart Lungs
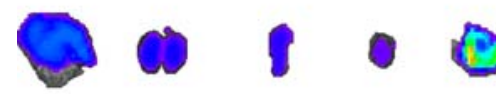

$1 \mathrm{~h}$
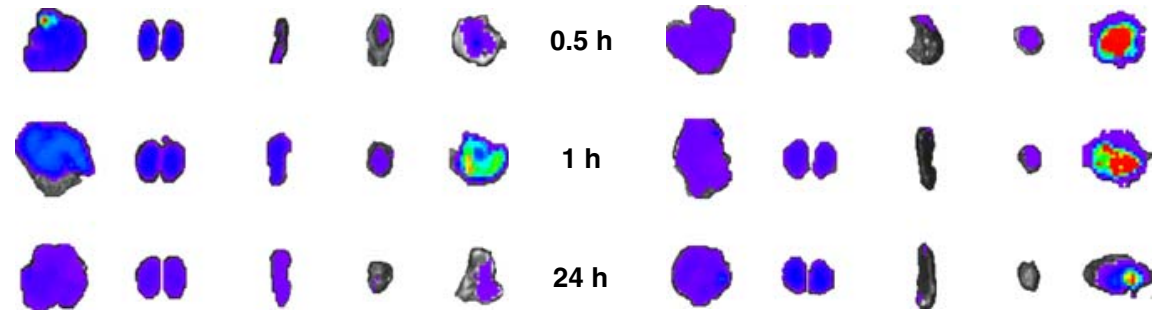

$24 \mathrm{~h}$

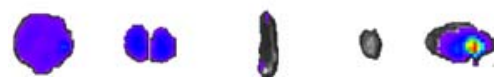

b

Liver

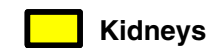

Kidneys $\quad \square$ Spleen

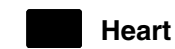

Heart $\square$ Lungs
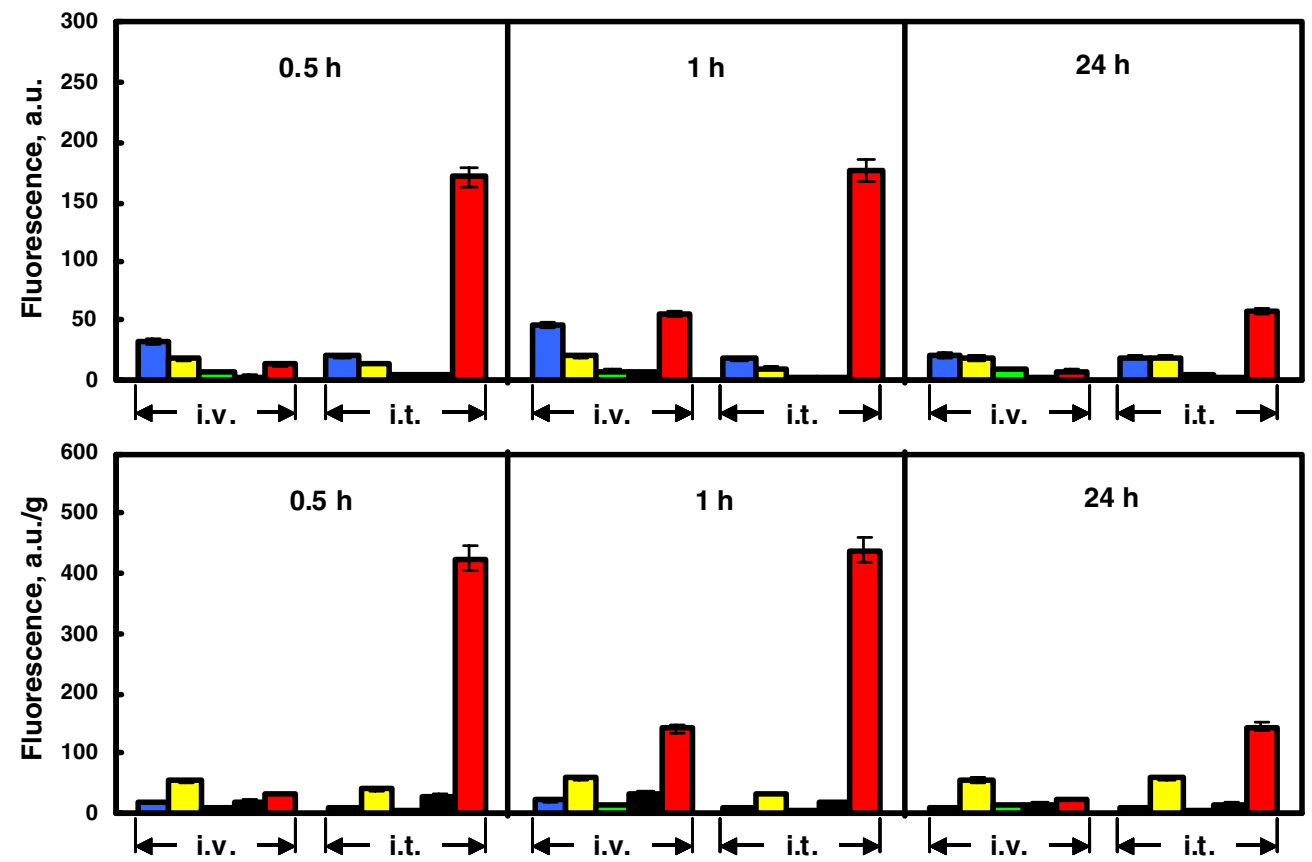

Fig. 4. Organ content of cationic liposomes. Liposomes were labeled with near infrared dye Cy5.5 and injected intravenously or intratracheally into the mice. The distribution of liposomes was measured using IVIS Xenogen imaging system. The upper panel (a) contains representative images of different organs excised $0.5,1$ and $24 \mathrm{~h}$ after injection. The intensity of fluorescence is expressed by different colors with blue color reflecting the lowest intensity and red color-highest intensity. The bottom panel (b) demonstrates average total content per an entire organ and concentration (per gram of organ weight) of labeled liposomes. Means \pm SD are shown.

Furthermore, liposomes modification with siRNA makes their appearance "fuzzy", thus hiding object topology.

Cellular Uptake of Liposomes, ASO, DOX, and siRNA. The delivery of ASO, DOX and siRNA by liposomes into cells was studied in vitro using confocal microscopy. A-549 human lung carcinoma cells were incubated with "neutral" liposomes containing ASO or DOX, cationic liposomes labeled with Cy5.5 or DOTAP/siRNA complexes and then intracellular liposomes distribution was examined. Results are shown on Fig. 2. As can be seen, after $24 \mathrm{~h}$ incubation, "neutral" and cationic liposomes were detected in the cellular cytoplasm and nucleus. Fluorescence of the payload of "neutral" liposomes (DOX or ASO) was also registered both in the cellular cytoplasm and nucleus. It is known that liposomes quench the fluorescence of any loaded agent (36). Therefore, the visualization of fluorescence strongly suggests that after $24 \mathrm{~h}$ of incubation ASO and DOX were completely released from liposomes and localized in the cellular plasma and nucleus. The results 
Neutral PEGylated Liposomes-Cy5.5
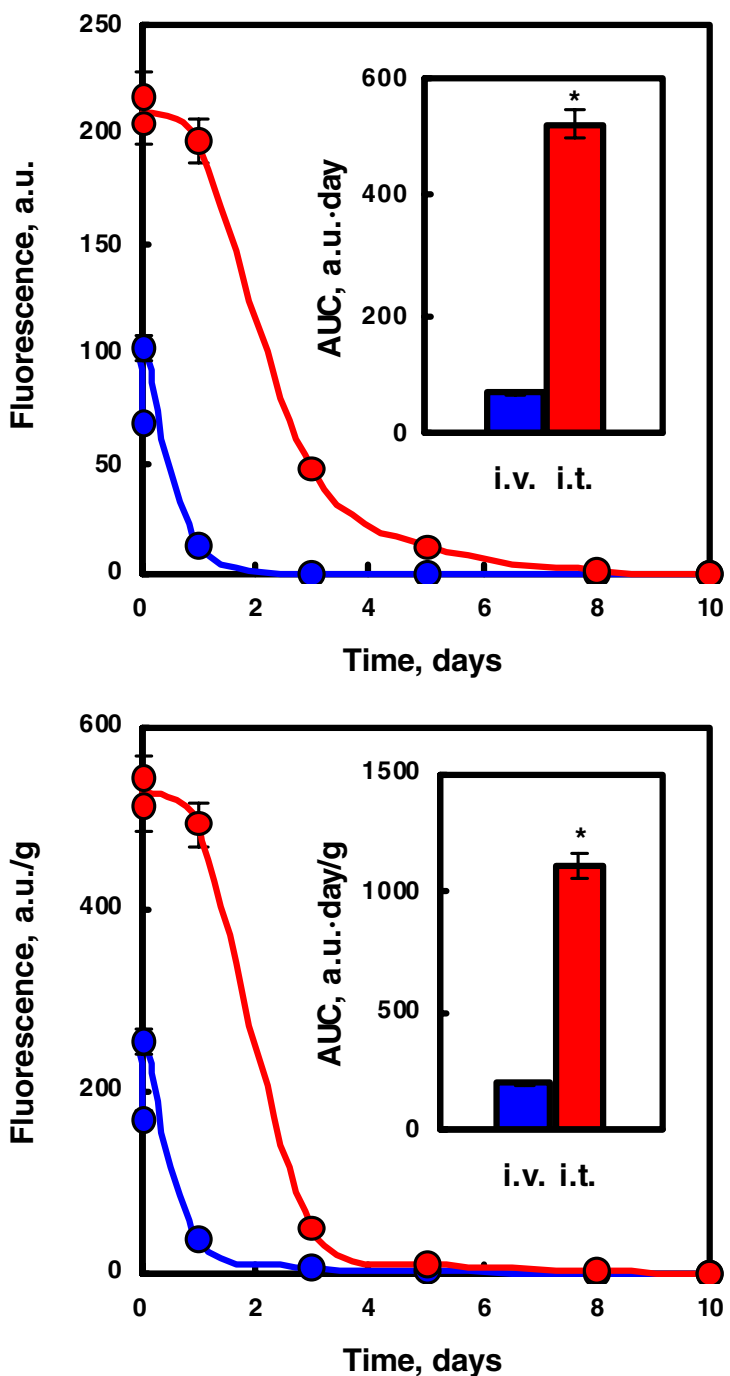

Cationic Liposomes-Cy5.5
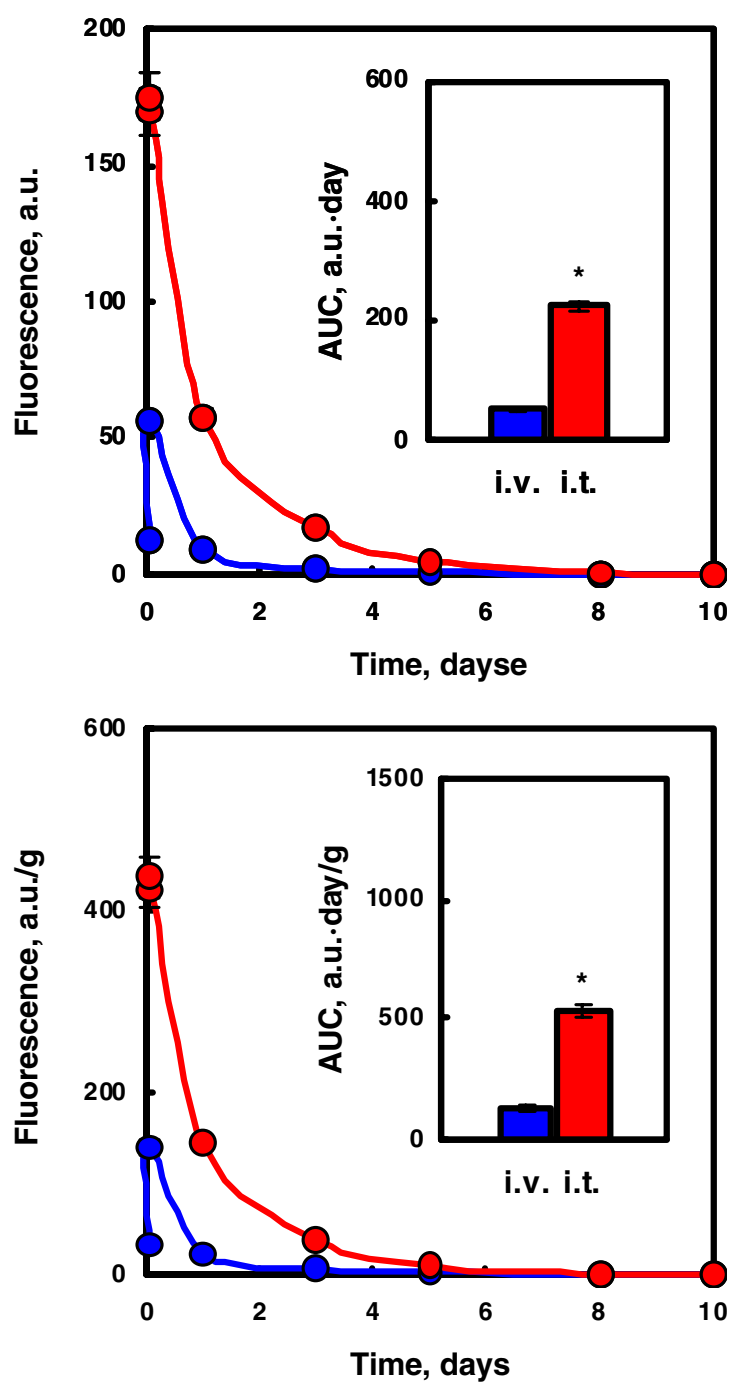

Fig. 5. Retention of different liposomes in lung tissues after intravenous (i.v.) and intratracheal (i.t.) instillation. The fluorescence of neutral PEGylated and cationic liposomes labeled with Cy5.5 was measured in the excised lungs within 10 days. An absolute and normalized per organ weight levels of fluorescence were plotted versus time and an area under the curve (AUC) was calculated. Means $\pm \mathrm{SD}$ are shown. $* P<0.05$ when compared with intravenous injection.

obtained also show that after $24 \mathrm{~h}$ of incubation, a substantial amount of complexes DOTAP/siRNA penetrated into cellular cytoplasm area (Fig. 2, bottom panel) despite their relatively big size $(>500 \mathrm{~nm})$. In order to prove that liposomes were not adhered on the cell surface but actually penetrated the cells, we analyzed their distribution in different cellular layers from the upper to the lower of the fixed cells ( $z$-sections, results are not presented). The data obtained show that the distribution of either small (100$140 \mathrm{~nm})$ "neutral" and cationic liposomes or large $(>500 \mathrm{~nm})$ DOTAP/siRNA complexes in cytoplasm and nucleus was very similar in different cell layers. Therefore, both systems can be used for delivery of therapeutic agents with biological activity into the cellular cytoplasm and nuclei.

Organ Content of "Neutral" and Cationic Liposomes After Intravenous and Intratracheal Instillation. Analysis of the total organ content of "neutral" and cationic liposomes revealed several common and distinct features (Figs. 3, 4, and 5). After intravenous (i.v.) injection the total content of both types of liposomes per an entire organ was highest in liver, kidneys, and lungs. However, the total amount of "neutral" liposomes retained in these organs was higher when compared with cationic liposomes. Intratracheal instillation (i.t.) of both types of liposomes substantially increased their total amount in entire lungs and decreased the content in other organs. Twenty four hours after i.t. injection, a substantially higher amount of labeled "neutral" liposomes per an entire organ was found in the lungs when compared with cationic liposomes. In addition, total content of liposomes in organs was normalized for organ weight. The concentrations of liposomes were highest in the kidneys and the lungs after intravenous injection. Intratracheal administration of liposomes limited the exposure of the kidneys and increased the concentration of liposomes in the lungs. Intra- 
Free DOX

a

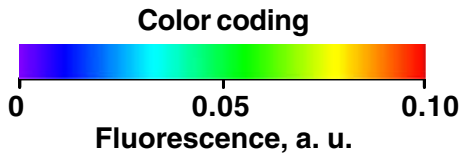

Intravenous injection

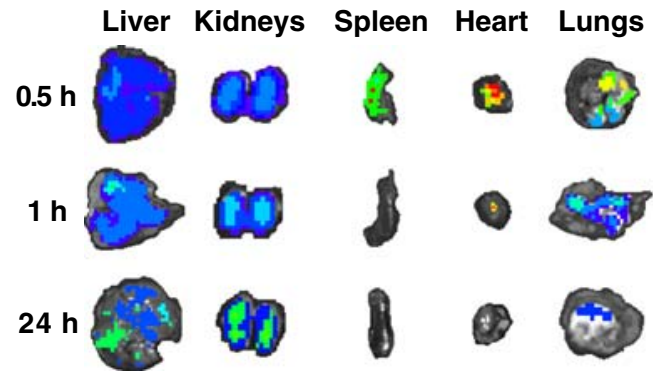

b $\quad \square$ Liver $\square$ Kidneys

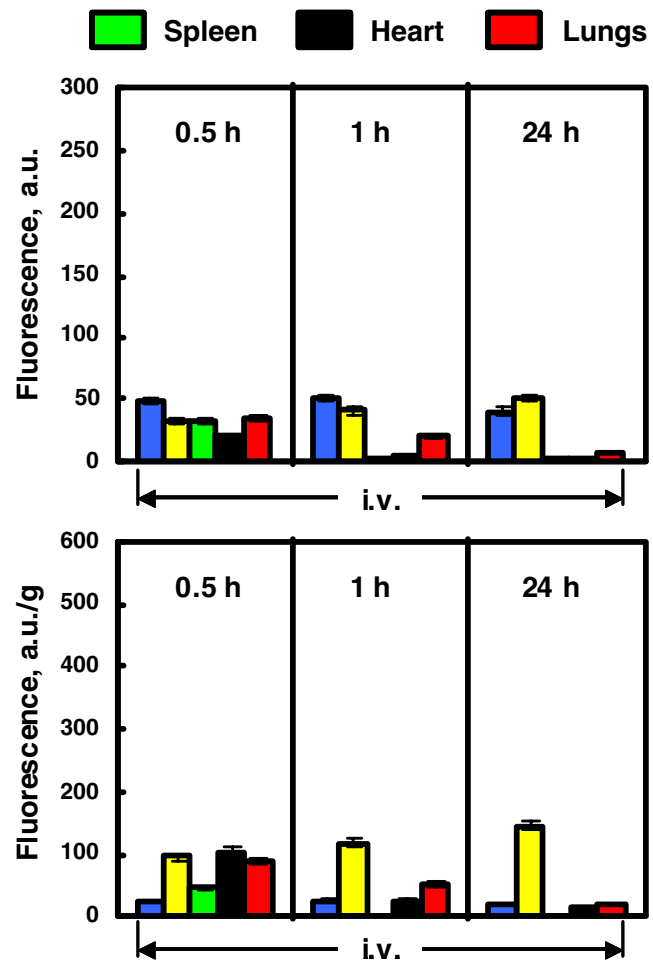

Fig. 6. Organ content of free doxorubicin $(D O X)$. DOX was injected intravenously into the mice. The distribution of DOX possessing intrinsic fluorescence was measured using the IVIS Xenogen imaging system. The upper panel (a) contains representative images of different organs excised $0.5,1$ and $24 \mathrm{~h}$ after injection. The intensity of fluorescence is expressed by different colors with blue color reflecting the lowest intensity and red-highest intensity. The bottom panel (b) demonstrates average total content per an entire organ and concentration (per gram of organ weight) of DOX. Means \pm SD are shown.

tracheal delivery of liposomes led to their much higher concentration in the lungs and retained this higher concentration for a much longer period of time when compared with intravenous injection (Fig. 5). It was found that liposomes completely eliminated from the lungs on the second-third days after intravenous injection. In contrast, a substantial concentration of liposomes was observed in the lungs during this period. The overall lung exposure calculated as an area under the curve (AUC) was 4 to 7.5 times higher after intratracheal instillation when compared with the intravenous injection. Therefore, intratracheal delivery of liposomes substantially increased the overall tissue exposure in the lungs and reduced the exposure and the peak tissue liposomal concentrations in other organs.

Organ Content of Free and Liposomal DOX. During the first $0.5 \mathrm{~h}$ after $i . v$. injection, a comparable total amount of DOX was found in all investigated organs (Fig. 6). However, at the later time-periods, the distribution was substantially changed. The highest total amount of DOX per an entire organ was found in the liver and kidneys, while the concentration of DOX was highest in the kidneys. Only trace amounts of DOX were found in the lungs $24 \mathrm{~h}$ after $i . v$. injection. Delivery of DOX by "neutral" liposomes substantially increased its total amount and concentrations in all studied organs (Figs. 7 and 8). However, the tendency in the dynamic of the organ concentration of DOX delivered by liposomes was similar to that of free DOX. During the first hour after its $i$.v. systemic delivery by liposomes, DOX was found predominately in the liver, kidneys, and lungs, and DOX concentration per gram of tissue was highest in the lungs and kidneys. The amount of DOX in the lungs declined $24 \mathrm{~h}$ after treatment. Intratracheal local delivery of liposomal DOX substantially changed the distribution of delivered DOX between the studied organs. After i.t. delivery, a predominant amount of the drug was found in the lungs during all studied time periods after injection. The analysis of accumulation of DOX normalized for organ weights showed that the tissue concentration of the cytotoxic drug in the lungs was elevated for a prolonged period of time and the peak tissue levels in the non-targeted tissues were reduced after intratracheal local delivery of liposomal DOX.

Organ Content of ASO and siRNA Delivered by "Neutral" and Cationic Liposomes After Intravenous and Intratracheal Injection. Organ content of ASO and siRNA delivered by "neutral" and cationic liposomes was similar to that of liposomal DOX (please compare Figs. 7, 8, and 9). Intravenous delivery led to the highest total content per an entire organ of ASO and siRNA in the liver, kidneys, and lungs. In most cases, $24 \mathrm{~h}$ after the i.v. injection the liposomal payloads were found mainly in the liver and kidney, while their amount retained in the lungs substantially decreased when compared with earlier periods after the treatment. It is interesting, that an amount of relatively large DOTAP/siRNA complexes $(>500 \mathrm{~nm})$ accumulated in the lungs was higher in the later stages of the experiment (1-24 h), while the quantity of smaller cationic liposomes without siRNA (100-140 nm) was higher in the earlier stages $(0.5-1 \mathrm{~h})$. It is possible that larger liposome/siRNA complexes require longer time for the degradation of an entire complex and siRNA release. Similar to liposomal DOX, after i.t. delivery, the highest concentrations of siRNA and ASO in all investigated time-points were registered in the lungs, while concentrations in nontargeted tissues were significantly lower. In contrast, $24 \mathrm{~h}$ 


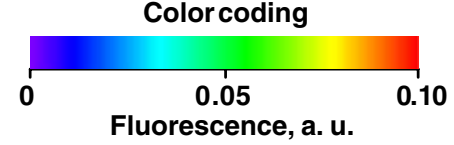

Intravenous injection

Intratracheal injections

Liver Kidneys Spleen Heart Lungs

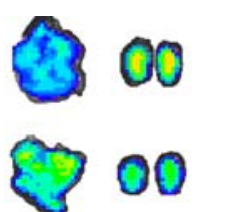

4 跑
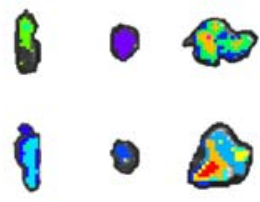

8 回

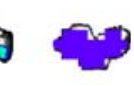

$0.5 \mathrm{~h}$

$1 \mathrm{~h}$

$24 \mathrm{~h}$
Liver Kidneys Spleen Heart Lungs
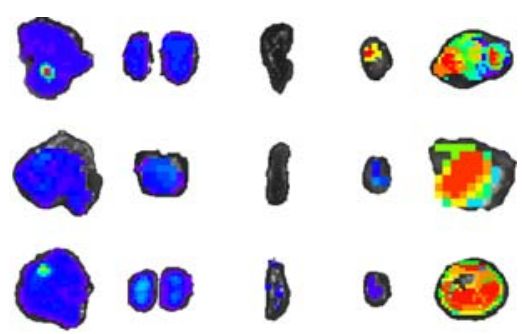

b

Liver

Kidneys

Spleen

Heart

Lungs
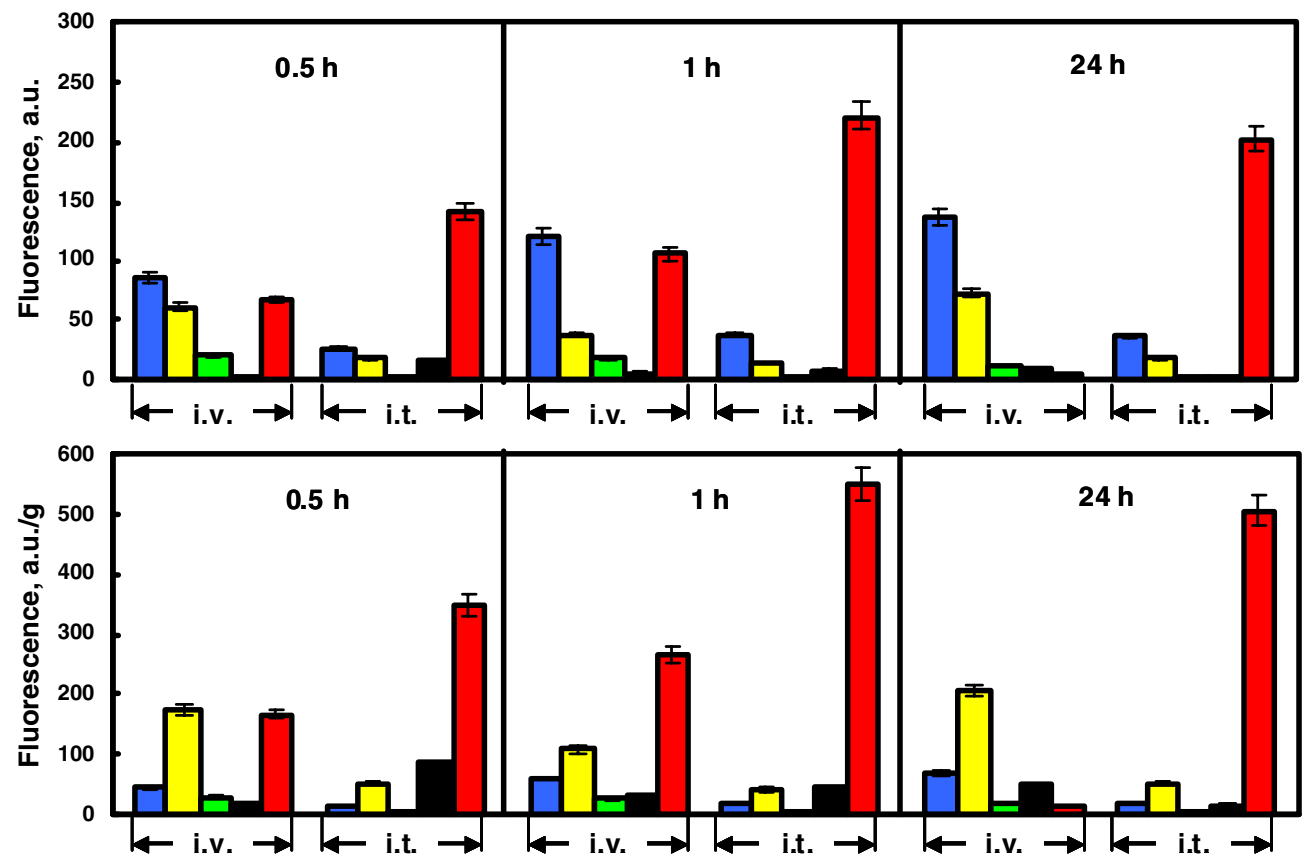

Fig. 7. Organ content of "neutral" liposomes containing doxorubicin $(D O X)$. PEGylated liposomes were delivered intravenously or intratracheally into the mice. Intrinsic fluorescence of DOX was measured using IVIS Xenogen imaging system. The upper panel (a) contains representative images of different organs excised $0.5,1$ and $24 \mathrm{~h}$ after injection. The intensity of fluorescence is expressed by different colors with blue color reflecting the lowest intensity and the red color reflecting the highest intensity. The bottom panel (b) demonstrates average total content per an entire organ and concentration (per gram of organ weight) of labeled liposomes. Means \pm SD are shown.

after i.v. injection, the highest concentrations of ASO as siRNA were found in the kidneys.

Intratracheal and Intravenous Treatment of Lung Tumor. Human lung tumor-bearing mice were injected with free DOX and liposomal DOX intravenously and intratracheally respectively. The results of these experiments are shown on the Fig. 10. It was found that treatment with intravenous injection of free DOX slightly decreased the number of cancer cells in the lungs (bar 2). Intravenous treatment with liposomal DOX led to a more substantial decrease in the number of tumor cells (bar 3). However, the most significant positive effect was found after the local delivery of liposomal DOX directly to the lungs by intratracheal injection (bar 4). In this case, after local intratracheal delivery of liposomal DOX, the number of cancer cells was decreased by $45 \%$ when compared with untreated tumor, by $38 \%$ when compared with treatment by intravenous injection of free DOX, and by $30 \%$ when compared with intravenous injection of liposomal DOX. 


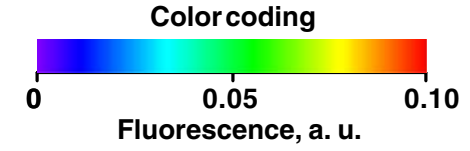

Intravenous injection

Intratracheal injections

Liver Kidneys Spleen Heart Lungs

Liver Kidneys Spleen Heart Lungs
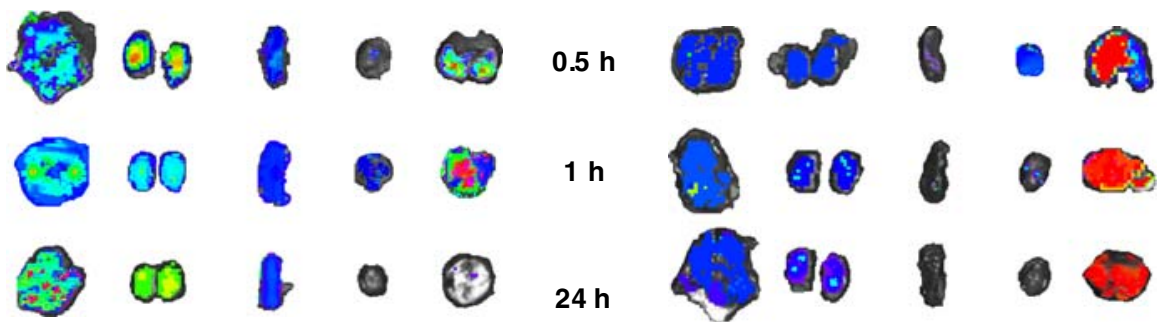

$24 \mathrm{~h}$

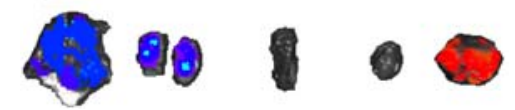

b

Liver

Kidneys

Spleen

Heart

Lungs
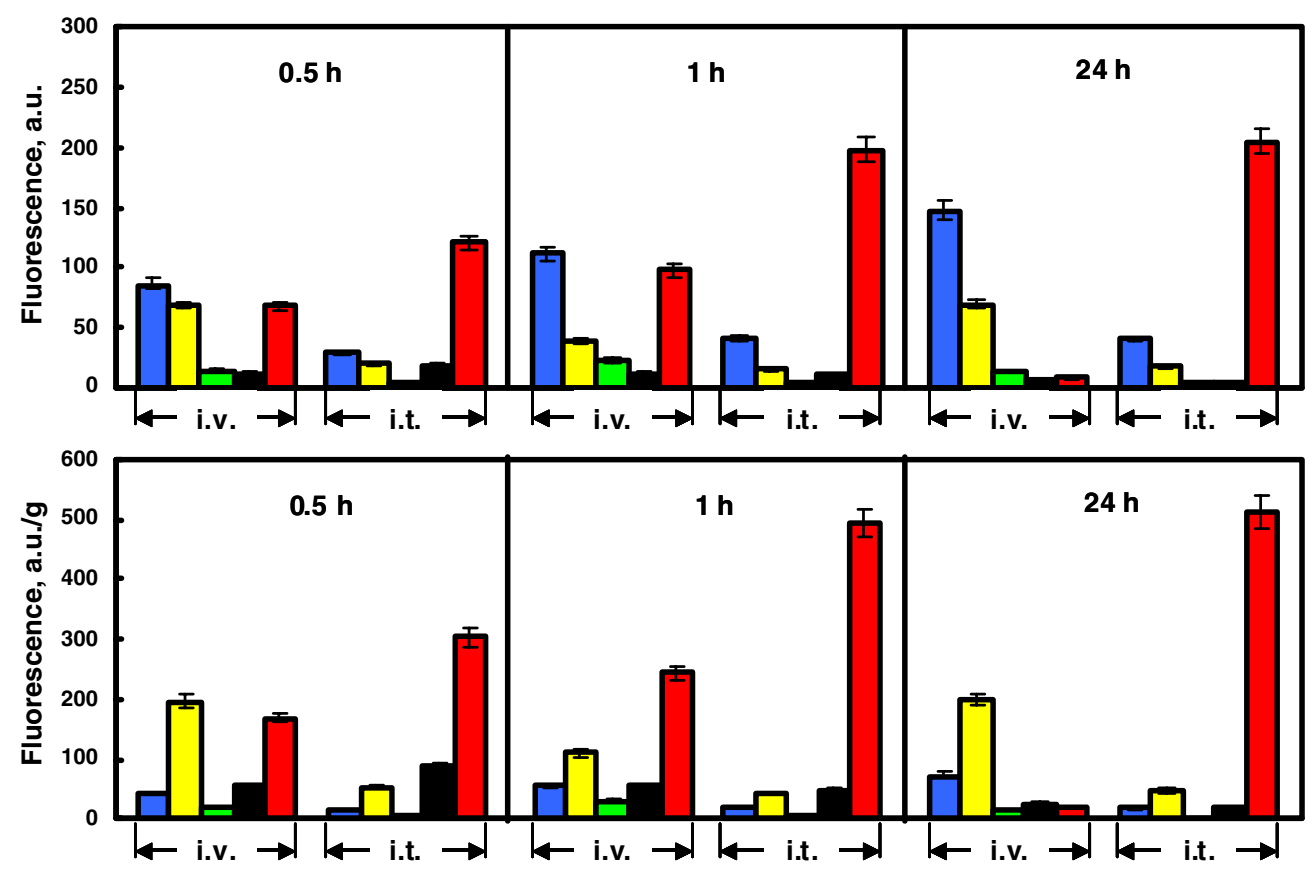

Fig. 8. Organ content of antisense oligonucleotides $(A S O)$ delivered by "neutral" liposomes. P-ethoxy neutral ASO were labeled with fluorescein isothiocyanate (FITC), encapsulated into "neutral" liposomes and delivered intravenously or intratracheally into the mice. The distribution of ASO was measured using the IVIS Xenogen imaging system. The upper panel (a) contains representative images of different organs excised $0.5,1$ and $24 \mathrm{~h}$ after injection. The intensity of fluorescence is expressed by different colors with blue color reflecting the lowest intensity and red color reflecting the highest intensity. The bottom panel (b) demonstrates average total content per an entire organ and concentration (per gram of organ weight) of labeled ASO. Means \pm SD are shown.

\section{DISCUSSION}

It is well known that liposomal drug formulations are more efficient in terms of their cellular internalization, specific activity, and adverse side effects when compared with free drugs $(28,37-43)$. Liposomes of different sizes and surface electric charges are currently being used for the delivery of encapsulated drugs or complexated large molecules. In addition to systemic intravenous injection, different routes of liposome administration including pulmonary have been proposed. While the high effectiveness of liposomal formulations is widely acknowledged, several aspects of liposomal drug delivery are not clearly understood. It is not apparent yet what is the optimum size and charge of 
a

siRNA-DY-547 Delivered by Cationic Liposomes
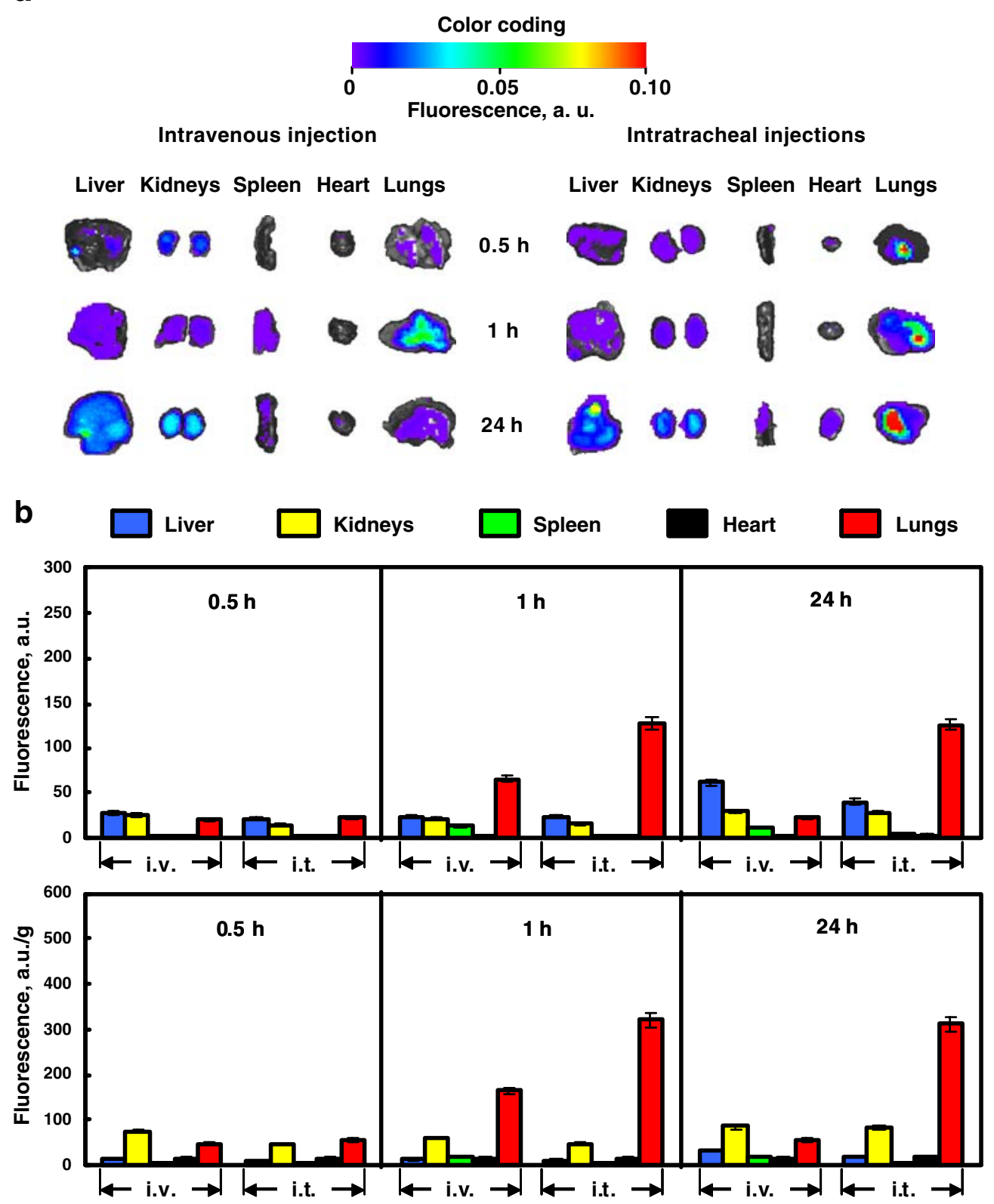

Fig. 9. Organ content of siRNA delivered by cationic liposomes. siRNA labeled with near infrared dye (siGLO red) was complexated with cationic liposomes and delivered intravenously or intratracheally into the mice. The distribution of siRNA was measured using IVIS Xenogen imaging system. The upper panel (a) contains representative images of different organs excised $0.5,1$ and $24 \mathrm{~h}$ after injection. The intensity of fluorescence is expressed by different colors with blue color reflecting the lowest intensity and red color reflecting the highest intensity. The bottom panel (b) demonstrates average total content per an entire organ and concentration (per gram of organ weight) of labeled siRNA. Means \pm SD are shown. liposomal drugs in terms of their successful intracellular uptake, organ retention, and treatment efficacy after administration via different routes. We address the problem in the present study by investigating in vitro cellular uptake and intracellular localization along with in vivo organ exposure after intravenous or intratracheal administration of small slightly negative ("neutral") and positively charged liposomes as well as their relatively large complexes with siRNA.
We used small "neutral" PEGylated and positively charged DOTAP liposomes. DOX and uncharged P-ethoxy ASO were used as a payload for neutral liposomes while siRNA was complexated to cationic liposomes. Atomic force microscopy and light scattering data showed that empty liposomes of both types represent homogenous spherical structures with diameter of 100-140 nm. Loading of "neutral" liposomes with DOX or neutral modified ASO did not change their average size and homogeneity. In contrast, 


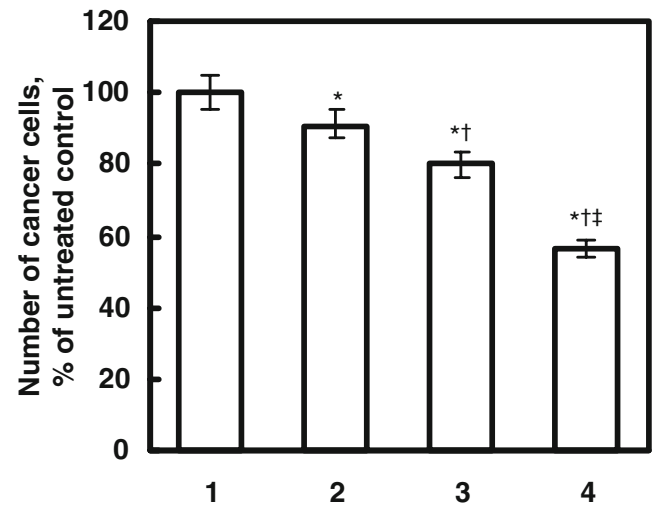

Fig. 10. Influence of intravenous free $D O X(2)$, intravenous liposomal DOX (3) and intratracheal liposomal DOX (4) delivery on the number of cancer cells in mice lungs. The number of cells was expressed as percent of untreated control (1). $* P<0.05$ when compared with untreated control. $\dagger P<0.05$ when compared with free DOX (i.v. injection). $\ddagger P<0.05$ when compared with intravenous liposomal DOX (i.v. injection).

complexation of cationic liposomes with siRNA led to dramatic (up to ten-times) increase in their diameter. Investigation of cellular internalization, localization, and distribution of liposomes showed that despite the differences in size, both small empty and loaded liposomes as well as relatively large complexes were able to deliver their payload to the cellular cytoplasm and even nuclei. Moreover, labeled "neutral" liposomes, as well as their payload, were found inside cellular nuclei. These data confirm our previous transmission electron and fluorescence microscopy findings for the nuclear penetration of conventional and "neutral" liposomes with average size of $100-200 \mathrm{~nm}$ both in vitro and in vivo $(28,31,44)$. It seems that, in contrast to small liposomes, relatively large DOTAP/siRNA complexes were not able to penetrate cellular nuclei. Therefore, liposomes and liposomal complexes of different sizes (100-1000 nm) used in this study were able to provide an efficient delivery of different biologically active substances, including antibiotics, antisense oligonucleotides, and small interfering RNA into the cellular cytoplasm.

Systemic administration of drugs, especially for treatment of lung diseases has several disadvantages including, but not limited to, unfavorable organ accumulation and decrease in specific drug activity as a result of drug deactivation during systemic circulation and high possibility of adverse side effects on healthy organs. Delivery of biologically active substances as liposomal prodrugs can partially improve the situation especially in case of using "neutral" "stealth" liposomes as carriers. However, our data showed that intratracheal administration of liposomes sustained higher concentrations of liposomes for up to three days and their payload in the lungs for at least $24 \mathrm{~h}$ after instillation. Once again, the size of liposomes and liposomal complexes with siRNA played a secondary role, while the route of administration served as the primary determinant of organ exposure by liposomes and their payload. Therefore, after the intratracheal delivery the tissue concentrations of liposomes and their payload in the lungs were elevated for a prolonged period of time and the peak tissue levels in the non-target tissues were reduced. These data clearly show potential advantages of local lung delivery of different liposomal therapeutics for the treatment of lung diseases and confirm that liposomes can be used for intratracheal delivery of drugs, antisense oligonucleotides, and siRNA into the lungs.

The efficient delivery of therapeutics to the site of action, however, does not automatically guarantee successful treatment of a disease. In order to confirm that intratracheal delivery of anticancer drugs can enhance the effectiveness of treatment of lung diseases, we used the orthotopic mice model of human lung cancer. Mice bearing human cancer cells in their lungs were treated with free and liposomal doxorubicin delivered intravenously and intratracheally. It was found that intratracheal treatment was much more efficient in limiting the growth of lung cancer cells in the lungs when compared with free and liposomal DOX delivered via the systemic route. Therefore, the present study showed advantages of local intratracheal delivery of liposomal drugs for the treatment of lung cancer when compared with systemic administration of the same drug.

\section{ACKNOWLEDGEMENTS}

The research was supported in part by NIH grants CA100098, CA111766, and CA074145 from the National Cancer Institute and by a LCD-23812-N grant from the American Lung Association of New Jersey.

Open Access This article is distributed under the terms of the Creative Commons Attribution Noncommercial License which permits any noncommercial use, distribution, and reproduction in any medium, provided the original author(s) and source are credited.

\section{REFERENCES}

1. American Lung Association: Research Awards Nationwide 2007-2008. http://www.lungusa.org/atf/cf/\%7B7a8d42c2-fcca4604-8ade-7f5d5e762256\%7D/RAN07-08.PDF (accessed 04/20/ 2008) (2008).

2. S. S. Dharap, Y. Wang, P. Chandna, J. J. Khandare, B. Qiu, S. Gunaseelan, P. J. Sinko, S. Stein, A. Farmanfarmaian, and T. Minko. Tumor-specific targeting of an anticancer drug delivery system by LHRH peptide. Proc. Natl. Acad. Sci. U. S. A. 102:12962-12967 (2005).

3. T. Minko, P. Kopeckova, and J. Kopecek. Efficacy of the chemotherapeutic action of HPMA copolymer-bound doxorubicin in a solid tumor model of ovarian carcinoma. Int. J. Cancer. 86:108-117 (2000).

4. M. Carpenter, M. W. Epperly, A. Agarwal, S. Nie, L. Hricisak, Y. Niu, and J. S. Greenberger. Inhalation delivery of manganese superoxide dismutase-plasmid/liposomes protects the murine lung from irradiation damage. Gene Ther. 12:685-693 (2005).

5. M. Chougule, B. Padhi, and A. Misra. Nano-liposomal dry powder inhaler of tacrolimus: preparation, characterization, and pulmonary pharmacokinetics. Int. J. Nanomedicine. 2:675-688 (2007).

6. M. B. Everhart, W. Han, K. S. Parman, V. V. Polosukhin, H Zeng, R. T. Sadikot, B. Li, F. E. Yull, J. W. Christman, and T. S. Blackwell. Intratracheal administration of liposomal clodronate accelerates alveolar macrophage reconstitution following fetal liver transplantation. J. Leukoc. Biol. 77:173-180 (2005).

7. A. Gautam, C. J. Waldrep, and C. L. Densmore. Delivery systems for pulmonary gene therapy. Am. J. Respir. Medicine. 1:35-46 (2002). 
8. U. Griesenbach, A. Chonn, R. Cassady, V. Hannam, C Ackerley, M. Post, A. K. Tanswell, K. Olek, H. O'Brodovich, and L. C. Tsui. Comparison between intratracheal and intravenous administration of liposome-DNA complexes for cystic fibrosis lung gene therapy. Gene Ther. 5:181-188 (1998).

9. L. M. Hoesel, M. A. Flierl, A. D. Niederbichler, D. Rittirsch, S. D. McClintock, J. S. Reuben, M. J. Pianko, W. Stone, H. Yang, M. Smith, J. V. Sarma, and P. A. Ward. Ability of antioxidant liposomes to prevent acute and progressive pulmonary injury. Antioxid. Redox Signal. 10:973-981 (2008).

10. J. F. Marier, J. Lavigne, and M. P. Ducharme. Pharmacokinetics and efficacies of liposomal and conventional formulations of tobramycin after intratracheal administration in rats with pulmonary Burkholderia cepacia infection. Antimicrob. Agents Chemother. 46:3776-3781 (2002).

11. T. Minko, A. Stefanov, and V. Pozharov. Selected contribution: lung hypoxia: antioxidant and antiapoptotic effects of liposomal alpha-tocopherol. J Appl Physiol. 93:1550-1560 (2002); discussion 1549 .

12. A. Samad, Y. Sultana, and M. Aqil. Liposomal drug delivery systems: an update review. Curr. Drug Deliv. 4:297-305 (2007).

13. R. A. Schwendener. Liposomes in biology and medicine. $A d v$. Exp. Med. Biol. 620:117-128 (2007).

14. T. M. Allen, P. Williamson, and R. A. Schlegel. Phosphatidylserine as a determinant of reticuloendothelial recognition of liposome models of the erythrocyte surface. Proc. Natl. Acad. Sci. U. S. A. 85:8067-8071 (1988).

15. R. T. Proffitt, L. E. Williams, C. A. Presant, G. W. Tin, J. A. Uliana, R. C. Gamble, and J. D. Baldeschwieler. Liposomal blockade of the reticuloendothelial system: improved tumor imaging with small unilamellar vesicles. Science. 220:502-505 (1983).

16. A. Gabizon, H. Shmeeda, and Y. Barenholz. Pharmacokinetics of pegylated liposomal doxorubicin: review of animal and human studies. Clin. Pharmacokinet. 42:419-436 (2003).

17. A. L. Klibanov, K. Maruyama, V. P. Torchilin, and L. Huang. Amphipathic polyethyleneglycols effectively prolong the circulation time of liposomes. FEBS Lett. 268:235-237 (1990).

18. D. Papahadjopoulos, T. M. Allen, A. Gabizon, E. Mayhew, K. Matthay, S. K. Huang, K. D. Lee, M.C. Woodle, D.D. Lasic, C. Redemann et al. Sterically stabilized liposomes: improvements in pharmacokinetics and antitumor therapeutic efficacy. Proc. Natl. Acad. Sci. U. S. A. 88:11460-11464 (1991).

19. R. Zeisig, K. Shimada, S. Hirota, and D. Arndt. Effect of sterical stabilization on macrophage uptake in vitro and on thickness of the fixed aqueous layer of liposomes made from alkylphosphocholines. Biochim. Biophys. Acta. 1285:237-245 (1996).

20. A. A. Gabizon, H. Shmeeda, and S. Zalipsky. Pros and cons of the liposome platform in cancer drug targeting. J. Liposome Res. 16:175-183 (2006).

21. H. D. Han, A. Lee, T. Hwang, C. K. Song, H. Seong, J. Hyun, and B. C. Shin. Enhanced circulation time and antitumor activity of doxorubicin by comblike polymer-incorporated liposomes. $J$. Control. Release. 120:161-168 (2007).

22. Y. L. Hao, Y. J. Deng, Y. Chen, K. Z. Wang, A. J. Hao, and Y. Zhang. In-vitro cytotoxicity, in-vivo biodistribution and antitumour effect of PEGylated liposomal topotecan. J. Pharm. Pharmacol. 57:1279-1287 (2005).

23. T. L. Hwang, W. R. Lee, S. C. Hua, and J. Y. Fang. Cisplatin encapsulated in phosphatidylethanolamine liposomes enhances the in vitro cytotoxicity and in vivo intratumor drug accumulation against melanomas. J. Dermatol. Sci. 46:11-20 (2007).

24. M. A. Behlke. Progress towards in vivo use of siRNAs. Mol. Ther. 13:644-670 (2006).

25. S. Betigeri, R. I. Pakunlu, Y. Wang, J. J. Khandare, and T. Minko. JNK1 as a molecular target to limit cellular mortality under hypoxia. Mol. Pharmacol. 3:424-430 (2006).
26. D. B. Fenske, A. Chonn, and P. R. Cullis. Liposomal nanomedicines: an emerging field. Toxicol. Pathol. 36:21-29 (2008).

27. J. Kunisawa, T. Masuda, K. Katayama, T. Yoshikawa, Y. Tsutsumi, M. Akashi, T. Mayumi, and S. Nakagawa. Fusogenic liposome delivers encapsulated nanoparticles for cytosolic controlled gene release. J. Control. Release. 105:344-353 (2005).

28. T. Minko, R.I. Pakunlu, Y. Wang, J.J. Khandare, and M. Saad. New generation of liposomal drugs for cancer. Anticancer Agents Med. Chem. 6:537-552 (2006).

29. R. I. Pakunlu, Y. Wang, W. Tsao, V. Pozharov, T. J. Cook, and T. Minko. Enhancement of the efficacy of chemotherapy for lung cancer by simultaneous suppression of multidrug resistance and antiapoptotic cellular defense: novel multicomponent delivery system. Cancer Res. 64:6214-6224 (2004).

30. V. P. Torchilin, T. S. Levchenko, R. Rammohan, N. Volodina, B. Papahadjopoulos-Sternberg, and G.G. D'Souza. Cell transfection in vitro and in vivo with nontoxic TAT peptide-liposome-DNA complexes. Proc. Natl. Acad. Sci. U. S. A. 100:1972-1977 (2003).

31. Y. Wang, M. Saad, R. I. Pakunlu, J. J. Khandare, O. B. Garbuzenko, A. A. Vetcher, V. A. Soldatenkov, V. P. Pozharov, and T. Minko. Non-viral nanoscale-based delivery of antisense oligonucleotides targeted to HIF1 alpha enhances the efficacy of chemotherapy in drug resistant tumor. Clin. Cancer Res. 14:3607-3616 (2008).

32. S. Zalipsky, M. Saad, R. Kiwan, E. Ber, N. Yu, and T. Minko. Antitumor activity of new liposomal prodrug of mitomycin $\mathrm{C}$ in multidrug resistant solid tumor: insights of the mechanism of action. J. Drug Target. 15:518-530 (2007).

33. P. Chandna, M. Saad, Y. Wang, E. Ber, J. Khandare, A. A. Vetcher, V. A. Soldatenkov, and T. Minko. Targeted proapoptotic anticancer drug delivery system. Mol. Pharmacol. 4:668-678 (2007).

34. O. Garbuzenko, S. Zalipsky, M. Qazen, and Y. Barenholz. Electrostatics of PEGylated micelles and liposomes containing charged and neutral lipopolymers. Langmuir. 21:2560-2568 (2005).

35. M. Jones. The surface properties of phospholipid liposome systems and their characterization. Adv. Colloid Interface Sci. 54:93-128 (1995).

36. Y. Tsukioka, Y. Matsumura, T. Hamaguchi, H. Koike, F. Moriyasu, and T. Kakizoe. Pharmaceutical and biomedical differences between micellar doxorubicin (NK911) and liposomal doxorubicin (Doxil). Jpn. J. Cancer Res. 93:1145-1153 (2002).

37. T. L. Andresen, S. S. Jensen, T. Kaasgaard, and K. Jorgensen. Triggered activation and release of liposomal prodrugs and drugs in cancer tissue by secretory phospholipase A2. Curr. Drug Deliv. 2:353-362 (2005).

38. A. Fahr, P. van Hoogevest, S. May, N. Bergstrand, and M. L. S. Leigh. Transfer of lipophilic drugs between liposomal membranes and biological interfaces: consequences for drug delivery. Eur. J. Pharm. Sci. 26:251-265 (2005).

39. R. D. Hofheinz, S. U. Gnad-Vogt, U. Beyer, and A. Hochhaus. Liposomal encapsulated anti-cancer drugs. Anticancer Drugs. 16:691-707 (2005)

40. P. Sapraand, and T. M. Allen. Ligand-targeted liposomal anticancer drugs. Prog. Lipid Res. 42:439-462 (2003).

41. V. P. Torchilin. Recent advances with liposomes as pharmaceutical carriers. Nat. Rev. Drug Discov. 4:145-160 (2005).

42. V. P. Torchilin. Recent approaches to intracellular delivery of drugs and DNA and organelle targeting. Annu. Rev. Biomed. Eng. 8:343-375 (2006).

43. V. P. Torchilin. Targeted pharmaceutical nanocarriers for cancer therapy and imaging. AAPS J. 9:E128-E147 (2007).

44. R. I. Pakunlu, Y. Wang, M. Saad, J. J. Khandare, V. Starovoytov, and T. Minko. In vitro and in vivo intracellular liposomal delivery of antisense oligonucleotides and anticancer drug. J Control. Release. 114:153-162 (2006). 